ORIGINAL PAPER

\title{
Application of immunohistochemistry for Detection OF METASTASES IN SENTINEL LYMPH NODES OF NON-ADVANCED BREAST CANCER PATIENTS
}

\author{
Tomasz Nowikiewicz ${ }^{1}$, Ewa Śrutek ${ }^{2}$, Wojciech Zegarski ${ }^{3}$
}

\author{
${ }^{1}$ Clinical Department of Breast Cancer and Reconstructive Surgery, Oncology Center, Bydgoszcz \\ ${ }^{2}$ Department of Pathology, Surgical Oncology Clinic Collegium Medicum Nicolaus Copernicus University, \\ Oncology Center, Bydgoszcz \\ ${ }^{3}$ Surgical Oncology Clinic Collegium Medicum Nicolaus Copernicus University, Oncology Center, Bydgoszcz
}

\begin{abstract}
Any diagnostic method for detection of occult metastases in sentinel lymph node (SLN) could change postoperative therapeutic management. In this study, we attempted to evaluate the usefulness of immunohistochemical (IHC) studies for histopathological SLN examination and their impact on the diagnosis of metastatic lesions.

Analyzed data concerned 1358 breast cancer patients referred for sentinel lymph node biopsy (SLNB) between 2004 and 2012 with particular emphasis on broadening the scope of postoperative SLN histopathological assessment to include IHC. Sentinel lymph node involvement was diagnosed in $22.2 \%$ of patients. Use of the IHC method facilitated detection of $21.4 \%$ of all diagnosed SLN lesions (9.9\% of macrometastases, $60.0 \%$ of micrometastases, $100 \%$ of isolated tumor cells). $61.6 \%$ sensitivity and $100 \%$ specificity were obtained in the group of patients who underwent intraoperative SLN histopathological examination.

Use of immunohistochemistry for diagnostics of sentinel lymph node metastases in patients with breast cancer enables detection of a greater proportion of metastases, thus modifying both surgical (SLN macrometastases) and adjuvant treatment. As compared to $\mathrm{pN} 0$ patients and those with a metastasis found in HE staining, in patients with a metastasis in the sentinel lymph node diagnosed in IHC studies, no statistically significant differences were found concerning the long-term results of the implemented treatment.
\end{abstract}

Key words: breast cancer, sentinel lymph node biopsy, metastases, immunohistochemistry.

\section{Introduction}

Sentinel lymph node biopsy (SLNB) is a very useful tool for proper assessment of clinical stage of the disease in non-advanced breast cancer patients. This method, following introduction of breast conserving therapy (BCT) by Veronesi [1], is the next step to limit the negative consequences of surgery. However, SLNB meets all requirements of safety and radicalization of the proposed treatment [2-7].
Based on the research of Mansel et al. and other authors, SLNB is the recommended method of treatment in breast cancer patients without clinically suspicious axillary lymph nodes. In contrast to axillary lymph node excision, SLNB may be associated with significantly fewer postoperative complications and, consequently, with better quality of life of patients after treatment [2-7].

Any diagnostic method allowing detection of previously unrecognized SLN metastases could change 
further therapeutic management. However, as a result of the need to continue surgical treatment (axillary lymphadenectomy) or to administer adjuvant therapies (chemotherapy, radiotherapy) the longterm effects of applied treatment may change.

Currently used methods of SLN pathological assessment in breast cancer patients usually utilize intraoperative examination of lymph nodes excised during SLNB (frozen sections stained with hematoxylin and eosin - HE). HE staining is also used in paraffin sections, which are used for final postoperative SLN verification.

Current recommendations of experts (St. Gallen conference - 03/2011) do not include additional application of immunohistochemical studies (IHC) for histopathological SLN verification [8]. However, the significance of IHC methods remains the subject of discussion and equivocal opinions. By providing the possibility of diagnosing SLN metastases (partially including lesions that do not exceed $2 \mathrm{~mm}$ ), they may directly influence further patient management.

The aim of the present study was to determine the usefulness of immunohistochemical techniques (IHC) in postoperative histopathological evaluation of SLNs. Our results may be taken into account in the discussion about the merits of routine use of IHC in pathological assessment of SLNs.

\section{Material and methods}

\section{The studied group of patients}

One thousand three hundred and fifty-eight nonadvanced breast cancer patients with clinically negative axillary nodes ( $\mathrm{cN} 0)$ treated in the period from January 2004 to June 2012 were referred for SLNB. Mean patient age was $56.6 \pm 9.5$ years (patients aged 23 to 83 years).

In order to detect SLNs, the treated patients underwent injection of both technetium-99-m radioisotope on albumin vehicle (Nanocol) with activity of 75-100 MBq and aniline dye solution (2.5\% Patent Blue-V dye) - a mixed isotope-dye method - or only one of the markers was used (isotope or dye). The SLN in question was identified in 1285 patients, and these data constituted the clinical material subjected to statistical analysis.

It was the surgeon performing SLNB who made the decision with regard to SLN identification and to the mode of examination of the excised lymph node (by immediate intraoperative examination or by examination in a regular mode).

Depending on the diagnostic method, the lymph node with the greatest radiomarker concentration (as well as other nodes - lymph nodes of the SLN group or post-SLN - with radioactivity count greater than one tenth of SLN radioactivity, according to the "rule of 10\%" established by Martin et al. [9]) as well as any other suspicious lymph node discovered during intraoperative examination was considered SLN. If the SLN could not be unequivocally identified or SLN metastases were found intraoperatively, then patients were subjected to axillary lymphadenectomy.

According to the American Joint Committee on Cancer classification (AJCC) $[10]$, nodal lesions were divided depending on size into metastases (larger than $2 \mathrm{~mm}$ - macrometastases), micrometastases (lesions larger than $0.2 \mathrm{~mm}$ and not exceeding $2 \mathrm{~mm}$ ) and isolated tumor cells (ITC - up to $0.2 \mathrm{~mm}$ ).

If macrometastases were found in SLNs postoperatively, then axillary lymphadenectomy was performed in those patients. Surgery was radicalized (axillary lymphadenectomy) in some cases of micrometastases depending on the patient's decision. Use of adjuvant systemic therapy was determined by the disease recurrence risk score (Goldhirsch criteria [11]) and by the expected sensitivity to hormone therapy (based on quantitative assessment of steroid receptors).

The influence of IHC testing on final detection rates of SLN metastases was investigated in the analyzed group of patients. The relationship between the number and size of excised SLNs and the presence of nodal lesions (or lack thereof) as well as the diagnostic method used in the case of the primary tumor was also evaluated. The final number of removed SLNs was determined on the basis of histopathological examination.

\section{Histopathological evaluation of sentinel lymph node}

Intraoperative examination consisted of microscopic evaluation of frozen tissue sections, taken from the largest section of the node, routinely stained with hematoxylin and eosin (HE staining). Moreover, longitudinal sections of the whole node were performed at the smallest possible intervals and subjected to careful macroscopic assessment. Cytological imprints (HE stained) were also made from each SLN cross-section.

Final pathological sentinel lymph nodes assessment (or regular histopathological examination) was performed on paraffin block sections. SLNs were fixed in $10 \%$ buffered formalin (24-48 h), cut along their long axis into two, three or four parts of 3 or $4 \mathrm{~mm}$ in thickness and embedded in paraffin. A $4 \mu \mathrm{m}$ -thick section was removed from each paraffin block with a microtome and stained with hematoxylin and eosin. If HE-stained sections showed no evidence of metastases, then in some patients two more sections were cut from the deeper portions of paraffin block and subjected to IHC testing with anti-cytokeratin antibodies. The pathologist examining the sections decided on performing such tests (two of five histopathologists who examined sections obtained from excised SLNs also conducted additional IHC studies). Sections were mounted onto SuperFrost $(+)$ 
slides and dried at $60^{\circ} \mathrm{C}$ for 2 hours. The samples were rehydrated and antigen was retrieved by using a PTlink appliance (Dako) in a high-pH buffer (9.0). In the rehydrated sections endogenous peroxidase was blocked with 3\% hydrogen peroxide for $10 \mathrm{~min}-$ utes. IHC tests were performed by means of the EnVision method, using primary antibodies: CK AE1/ AE3 (Dako, M3515, at dilution $1:$ 100) and CK 7 (Dako, M7018, 1 : 100). The slides were incubated with the primary antibody for 30 minutes. Diaminobenzidine (DAB) was used as a chromogen for visualization and hematoxylin for staining of the nuclei. Sections of breast carcinoma known to be positive for CK 7 and CK AE1/AE3 were used as positive controls. Staining of both cytoplasm and cell membrane in cells exhibiting malignant morphology consistent with that of the primary tumor was considered a positive reaction. Morphological analysis allowed exclusion of irrelevant staining of macrophages or of endothelial cells in some cases.

\section{Statistical analysis}

In order to describe the data, tables were used showing the number and percentage distribution of groups as well as their means and standard deviations. A nonparametric Mann-Whitney $U$ test (Wilcoxon test) was used for statistical analysis of obtained results to assess differences with regard to one feature between two groups with non-normal distributions. Statistical significance of the differences between compared groups of data was achieved with $\mathrm{p}$ values below 0.05 .

The diagnostic value of intraoperative evaluation of SLNs was determined by assessing sensitivity (proportion of true positive to the sum of true positive and false negative results) and the specificity (proportion of true negative to the sum of true negative false positive results) of the test.

\section{Results}

Among 1285 patients in whom SLNs were identified, lymph node lesions were diagnosed in 285 subjects (in $77.9 \%$ they were macrometastases (222/285), in $21.1 \%$ micrometastases $(60 / 285)$, and in $1.1 \%$ ITC (3 patients)).

Examination of HE-stained sections (made during the SLNB or coming from paraffin blocks) gave a positive result in $17.4 \%$ of cases. HE sections showed no evidence of metastases in $50.2 \%$ of patients (533/1061) and IHC tests were additionally performed. Extending the scope of diagnostic methods made it possible to demonstrate the presence of metastases of SLNs in another 61 patients (detailed data are presented in Fig. 1).

Use of the IHC method facilitated diagnosis in $21.4 \%$ of all identified SLN metastases, and it in- creased the total number of diagnosed SLN metastases by over $27 \%$ (detailed data on the subject of detected macro- and micrometastases are included in Table I; Fig. 2 presents a microscopic image of a lymph node with a non-diagnosed metastasis on routine $\mathrm{HE}$ staining; Fig. 3 - metastases on IHC stain CK7).

Among the 61 patients mentioned above, the primary lesions were invasive in almost all cases (an intraductal carcinoma with diffuse type of growth was diagnosed in 1 patient only). Unequivocal indications for SLNB radicalization existed in 22 cases (patients with macrometastases). In 5 patients from this group, histopathological assessment of specimens after lymphadenectomy revealed metastases to other lymph nodes. In the remaining group of 39 patients with micrometastases or ITC, surgical treatment was continued (with subsequent systemic therapy in 27 cases), or adjuvant therapy alone was used (12 patients).

Detection of metastases due to IHC changed the risk of cancer recurrence in every fifth patient. Almost half of the cases (29/61) required modification of adjuvant therapy - administration of chemotherapy. A summary of SLN(+) patients' data is presented in Table II.

In the great majority of cases (91.2\%) intraoperative histopathological SLN assessment was performed during SLNB. In the remaining 113 subjects SLNs were examined in a regular manner. In 157 patients metastases were diagnosed by intraoperative assessment (true positive results), while in 98 patients intraoperative evaluation provided false negative results. There were no false positive diagnoses. According to the analysis, intraoperative SLN evaluation was characterized by $61.6 \%$ sensitivity and $100 \%$ specificity - for all types of lesions.

In the case of micrometastases, sensitivity of intraoperative SLN assessment was $11.3 \%$, and $75.9 \%$ for macrometastases (specificity of the test was $100 \%$ for both types of metastases).

Axillary lymphadenectomy was performed in all patients with macrometastases. In the group with micrometastases or ITC, $74.6 \%$ of patients $(47 / 63)$ underwent axillary lymphadenectomy. The remaining 16 patients came under strict postoperative supervision, including clinical assessment and ultrasound examination of the axilla at 3-month intervals.

Postoperative follow-up of patients treated with SLNB (observation period of 24 to 108 months) allowed diagnosis of 10 cases of disease recurrence (in the form of axillary recurrence or disease dissemination). All observed cases of treatment failure involved SLN(-) patients. No recurrences or neoplastic dissemination were observed in the group of patients with metastases in SLNs (diagnosed by IHC or HE stain) regardless of the type of adjuvant treatment (Table III contains detailed data). 


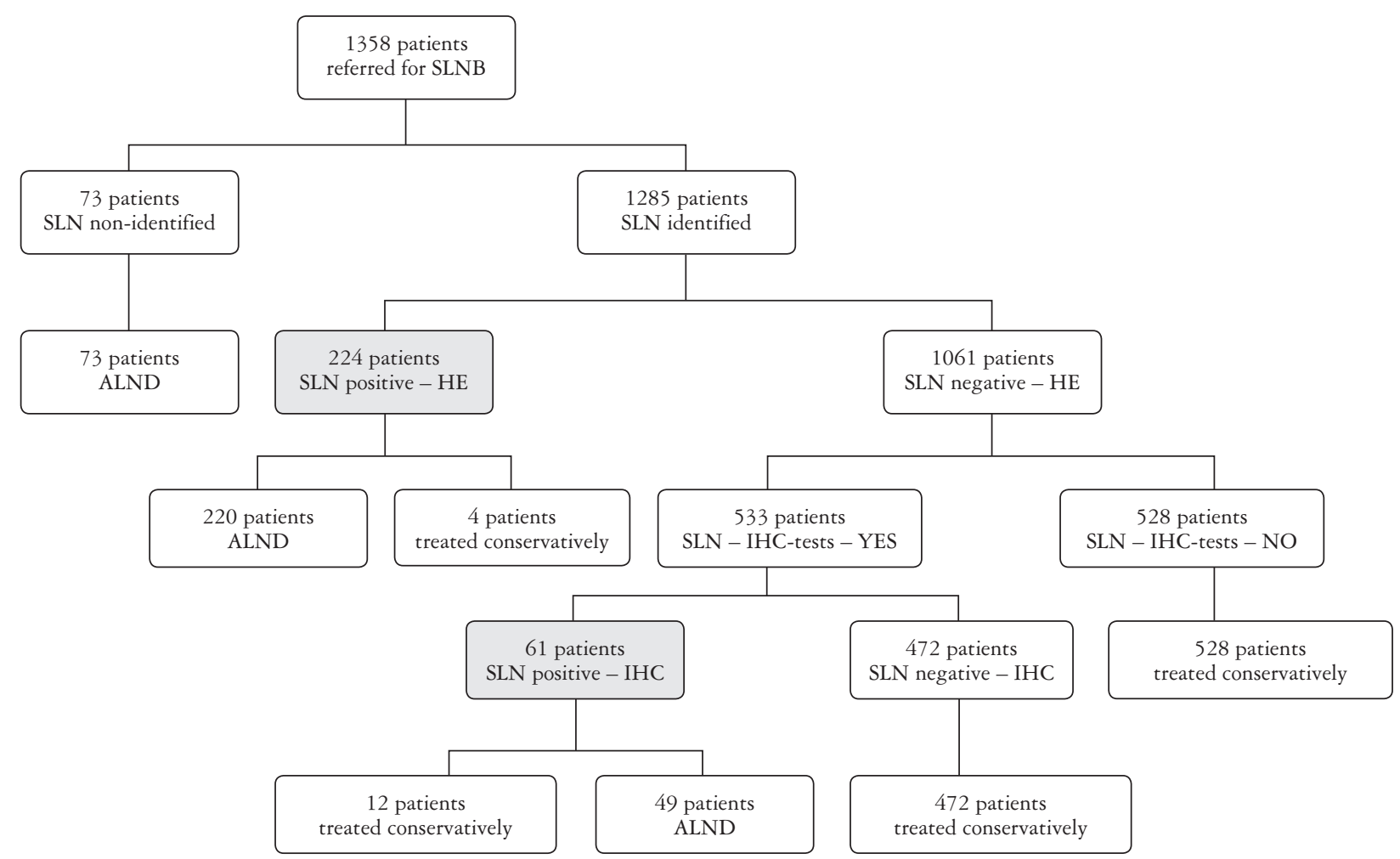

Fig. 1. Patients referred for sentinel lymph node biopsy - metastatic changes identified in sentinel lymph node and methods of histopathological assessment

Table I. Metastases diagnosed in sentinel lymph nodes

\begin{tabular}{|c|c|c|c|c|}
\hline Metastases in SLN & $\begin{array}{c}\text { Metastases IN SLN - } \\
\text { HE STAIN } \\
{[\text { A }](\%)}\end{array}$ & $\begin{array}{c}\text { Metastases IN SLN } \\
\text { - IHC } \\
{[\mathrm{B}](\%)}\end{array}$ & $\begin{array}{c}\text { Total NUmber of } \\
\text { METASTASES IN SLN } \\
{[\mathrm{A}+\mathrm{B}]}\end{array}$ & $\begin{array}{c}\text { INCREASE IN NUMBER } \\
\text { OF METASTASES DUE } \\
\text { TO USE OF IHC } \\
{[\mathrm{B} / \mathrm{A}](\%)}\end{array}$ \\
\hline Macrometastases & $200(90.1)$ & $22(9.9)$ & 222 & 11.0 \\
\hline Micrometastases & $24(40.0)$ & $36(60.0)$ & 60 & 150.0 \\
\hline ITC & $0(0.0)$ & $3(100.0)$ & 3 & \\
\hline Total & 224 & 61 & 285 & 27.2 \\
\hline
\end{tabular}

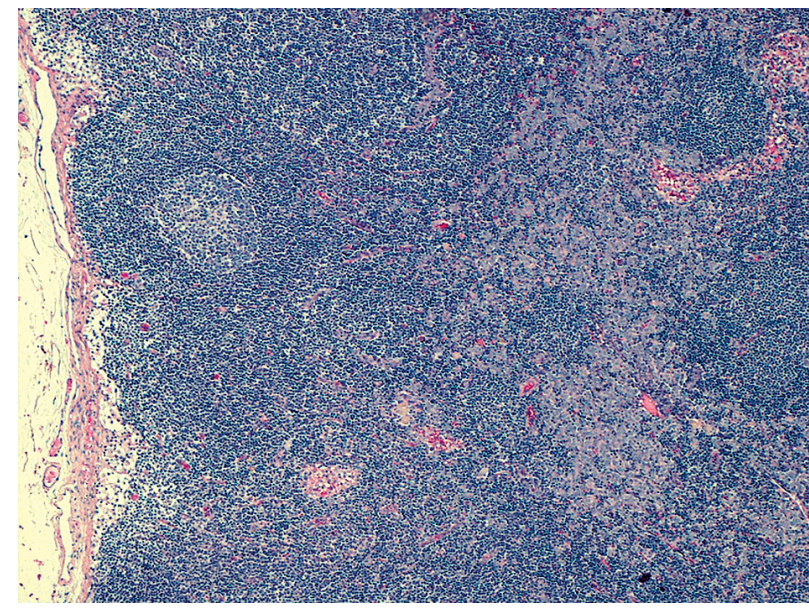

Fig. 2. Lymph node - routine HE staining

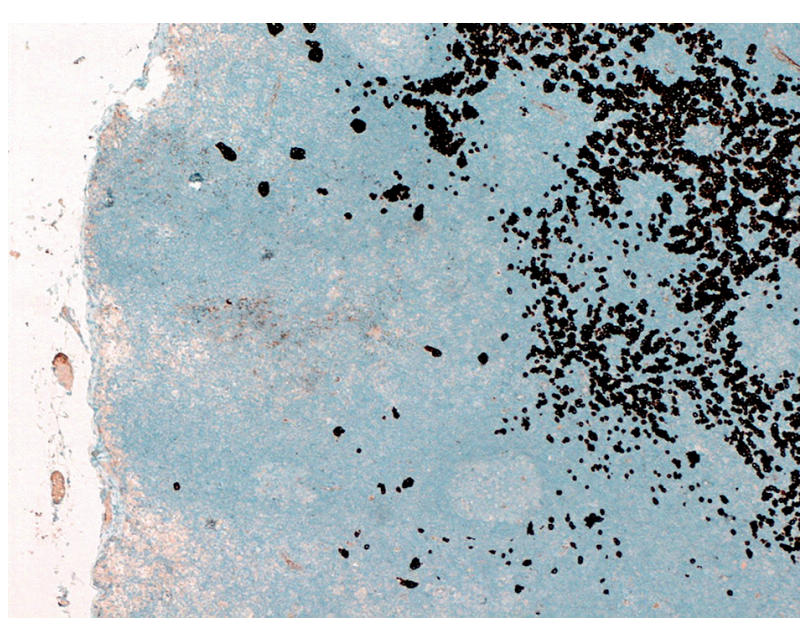

Fig. 3. The same lymph node, cells of invasive lobular carcinoma - metastases on IHC stain CK7 
Table II. Patients with metastases in sentinel lymph nodes diagnosed by immunohistochemical examination and hematoxylin-eosin stain

\begin{tabular}{|c|c|c|c|}
\hline & $\begin{array}{c}\mathrm{SLN}(+)-\mathrm{IHC} \\
\mathrm{N}=61(\%)\end{array}$ & $\begin{array}{c}\mathrm{SLN}(+)-\mathrm{HE} \\
\mathrm{N}=224(\%)\end{array}$ & $\mathrm{P}$ \\
\hline \multicolumn{4}{|l|}{ Age: } \\
\hline$<40$ years & $2(3.3)$ & $15(6.7)$ & ns \\
\hline $40-60$ years & $39(63.9)$ & $144(64.3)$ & ns \\
\hline$>60$ years & $20(32.8)$ & $65(29.0)$ & ns \\
\hline $\mathrm{pT} 1$ & $44(72.1)$ & $136(60.7)$ & $<0.05$ \\
\hline $\mathrm{pT} 2$ & $16(26.2)$ & $85(37.9)$ & ns \\
\hline pT3 & $1(1.6)$ & $3(1.3)$ & ns \\
\hline \multicolumn{4}{|l|}{ histological type of the tumor: } \\
\hline - invasive ductal carcinoma & $48(78.7)$ & $204(91.1)$ & $<0.05$ \\
\hline - invasive lobular carcinoma & $12(19.7)$ & $16(7.1)$ & $<0.05$ \\
\hline - other invasive & $1(1.6)$ & $4(1.8)$ & ns \\
\hline G1 & $8(13.1)$ & $17(7.6)$ & ns \\
\hline G2 & $44(72.1)$ & $143(63.8)$ & ns \\
\hline G3 & $7(11.5)$ & $58(25.9)$ & $<0.05$ \\
\hline nd & $2(3.3)$ & $6(2.7)$ & ns \\
\hline ER positive & $58(95.1)$ & $188(83.9)$ & $<0.05$ \\
\hline ER negative & $3(4.9)$ & $36(16.1)$ & ns \\
\hline HER2 positive & $2(3.3)$ & $35(15.6)$ & ns \\
\hline HER2 negative & $58(95.1)$ & $188(83.9)$ & $<0.05$ \\
\hline nd & $1(1.6)$ & $1(0.4)$ & ns \\
\hline ALND & $49(80.3)$ & $220(98.2)$ & $<0.05$ \\
\hline treated conservatively & $12(19.7)$ & $4(1.8)$ & $<0.05$ \\
\hline nSLN metastases & $8(13.1)$ & $75(33.5)$ & $<0.05$ \\
\hline
\end{tabular}

SLN (+) - sentinel lymph node metastases; T - size of primary tumor; $n d$ - no data; $G$ - assessment of malignancy degree (grading); HER2 - HER2 receptor; $E R$ - estrogen receptor; ALND - axillary lymph node dissection; nSLN - non-sentinel lymph nodes; ns - no statistical significance; $p$ - level of significance $p$

Table III. Disease recurrences observed in patients treated with SLNB

\begin{tabular}{ccccc}
\hline TyPe Of RECURRENCE & $\begin{array}{c}\text { SLN }(-)-\text { HE } \\
\mathbf{N}=528(\%)\end{array}$ & $\begin{array}{c}\text { SLN }(-)-\text { IHC } \\
\mathbf{N}=472(\%)\end{array}$ & $\begin{array}{c}\text { SLN }(+)-\text { HE } \\
\mathbf{N}=224(\%)\end{array}$ & $\begin{array}{c}\text { SLN }(+)-\text { IHC } \\
\mathbf{N}=61(\%)\end{array}$ \\
\hline Axillary fossa recurrence & $2(0.38)$ & $2(0.42)$ & $0(0)$ & $0(0)$ \\
\hline Distant metastases & $1(0.19)$ & $1(0.21)$ & $0(0)$ & $0(0)$ \\
\hline TOTAL & $3(0.57)$ & $3(0.64)$ & $0(0)$ & $0(0)$ \\
\hline
\end{tabular}

\section{Discussion}

Involvement of lymph nodes excised during SLNB was diagnosed in 285 of the studied cases. Use of cytokeratin IHC in postoperative histopathological evaluation allowed detection of occult metastases. In the majority of cases, this additional diagnosis involved micrometastases.

By extending the methodology of pathological SLN verification, it was possible to detect $21.4 \%$ of all metastases in operated patients. Similar results were obtained by Cserni et al. [4]. Their multicenter study summarized the data on treatment of 2929 patients with non-advanced breast cancer from 14 oncological centers around Europe. Additional application of IHC methods enabled detection of every fifth SLN metastasis (20.2\%). The total percentage of SLN metastases in the studied group was 21.9\% (the analysis concerned patients with primary breast cancer not larger than $15 \mathrm{~mm}$ ). 
According to reports by other authors, widening the scope of SLN assessment made it possible to diagnose up to $32.3 \%$ (Funasako et al. - 16.2\% [12], Giobuin et al. - 18.8\% [13], Carvalho et al. $-32.3 \%$ [14]) of all metastases (for the total number of SLN metastases diagnosed in the respective studies $18.1 \%, 24.2 \%$ and $25.7 \%$ ).

Mansel et al. [2] and Krag et al. [6] presented a different diagnostic strategy. They did not use IHC reactions for evaluation of SLNs removed during SLNB. In the first study, Mansel abandoned the intraoperative assessment of SLNs. Both studies involved large groups of patients -495 and 5611 subjects without clinical signs of axillary lymph node involvement and without restrictions with regard to the primary tumor size. Nevertheless, the number of lymph node metastases $-25.7 \%$ [2] and $28.9 \%$ [6] respectively was not smaller than with the results obtained using IHC methods.

Micrometastases and ITC predominated among lymph node lesions diagnosed through IHC (39/61). However, identification of a significant number of metastases larger than $2 \mathrm{~mm}(9.9 \%)$ is worth noting. Giobuin et al. [13] and Choi et al. [15] presented different results. In their studies, all diagnosed macrometastases were detected using routine staining methods (HE).

In our material, presence of metastases diagnosed through IHC did not change the risk of cancer recurrence in most patients. However, in almost every second patient it determined the kind of necessary adjuvant systemic therapy. In comparison to $\mathrm{pN} 0$ and patients with metastases in SLN diagnosed by $\mathrm{HE}$ stain $[\mathrm{SLN}(+)$-HE patients], there were no statistically significant differences with regard to therapeutic failure in the group with metastases in SLN diagnosed by IHC [SLN(+)-IHC patients]. Determination of long-term results of oncological treatment will be possible after a longer period of observation.

As compared to the SLN(+)-HE group, female SLN(+)-IHC patients showed more favorable prognostic factors. That concerned the size of the primary tumor, based on a histopathological examination (especially in case of a statistically significantly higher rate of $\mathrm{pT} 1$ lesions). A similar correlation was found in the case of estrogen receptors present in the tumor cells and no HER 2 receptor ( $95.1 \%$ vs. $83.9 \%)$. Moreover, patients from the SLN(+)-IHC group revealed a significantly lower rate of tumor lesions of high histological grade (G3) (11.5\% vs. $25.9 \%)$ and rate of metastatic lesions diagnosed in nonsentinel lymph nodes $(13.1 \%$ vs. $33.5 \%)$. In the latter case, concerning patients subjected to adjuvant axillary lymph node dissection (ALND), in five SLN(+)-IHC female patients there was a metastasis found in the sentinel lymph node exceeding $2 \mathrm{~mm}$ in size, and in a further 3 female patients there was a micrometastasis.
From the observations of Carvalho et al. it follows that only in the case of micrometastases present in sentinel lymph nodes, diagnosed in routine HE staining, can metastases be expected in subsequent lymph nodes of the axillary fossa. In the analyzed study they were found in $24 \%$ of $\mathrm{pN} 1 \mathrm{mi}$ patients and they concerned only SLN(+)-HE female patients [16].

According to Pugliese et al., identification of metastases through IHC is a negative prognostic factor and it is associated with increased necessity of adjuvant chemotherapy administration [17]. On the basis of over five-year follow-up of clinical material, the authors did not find differences concerning either the total survival time or the recurrence-free time as compared to the control group without metastases.

In our study, intraoperative histological SLN evaluation performed in most patients allowed us to detect existing nodal metastases only in some cases of nodal lesions. The overall sensitivity of intraoperative SLN examination (61.6\%) reached distinctly different values for metastases of different sizes. The difference between the results was statistically significant $(p<0.001)$. Effectiveness of intraoperative SLN frozen section evaluation described by Geertsema et al. [18] was slightly lower than ours. Performing intraoperative SLN assessment on each of 879 operated patients, they achieved $57.0 \%$ sensitivity. Complementing verification of HE-stained paraffin block sections with IHC facilitated correct diagnosis in the following 151 cases.

Carvalho et al. [14] obtained slightly higher sensitivity $(67.7 \%)$ of intraoperative SLN assessment. Tille et al. [19] presented even more favorable results. In the analyzed group of patients, sensitivity of the method in detecting macrometastases was $83.3 \%$ and $40 \%$ for micrometastases. There were no false positive results (100\% specificity for frozen sections made during SLNB).

Apart from the commonly used methods of pathological SLN assessment, there are also less popular diagnostic tools. Molecular methods can be applied for SLN verification. For example, GeneSearch BLN Assay test belonging to this group of diagnostic methods is based on quantitative measurement of cytokeratin 19 and mammaglobin expression. The test is designed for intraoperative use and takes about $30 \mathrm{~min}-$ utes on average (comparable with the time needed for immediate assessment of frozen sections). It utilizes polymerase chain reaction (reverse transcriptase-polymerase chain reaction - RT-PCR). Using the RT-PCR method for evaluation of SLNs in their prospective analysis, Blumencranz et al. obtained 98\% sensitivity for macrometastases, $57 \%$ sensitivity for micrometastases and $88 \%$ specificity for both types of lesions [20]. The authors also noted the possibility of false positive results ( $4 \%$ in their experiments). Funasako et al. obtained very similar results for detecting all me- 
tastases $(95.7 \%$ sensitivity for metastases larger than $2 \mathrm{~mm}, 60 \%$ for micrometastases and $55.6 \%$ for ITC) [12]. Mansel et al. reported $88.9 \%$ sensitivity using GeneSearch BLN Assay [21].

Another practical application of molecular analyses is the OSNA test (one-step nucleic acid amplification), which measures the amount of cytokeratin 19 mRNA. Using OSNA, Tsujimoto et al. achieved 98.2\% concordance with the result obtained from standard SLN assessment including intraoperative evaluation of frozen preparations and postoperative examination of material stained with $\mathrm{HE}$ and using IHC methods [22]. Evaluation of PTEN (phosphatase and tension homolog) and VEGF (vascular endothelial growth factor) expression may also serve as a tool to detect micrometastases. According to Zhu et al., the loss of PTEN expression with VEGF overexpression is very strongly correlated with the presence of SLN micrometastases ( $\mathrm{p}=0.001, \mathrm{r}=0.446$ ) in breast cancer patients [23].

Authors from the academic centre in Cracow are of a different opinion [24]. Their research indicates that breast carcinomas and their corresponding axillary lymph node metastases along with clinical stage of disease and the size of the primary tumor were not correlated with VEGF accumulation.

Among prognostic factors indicating a high correlation with possible unsatisfactory distant treatment results of breast cancer patients (including presence of metastases in regional lymph nodes), Gudlaugsson et al. specify mitotic activity index (MAI), phosphohistone H3 (PPH3) and Ki-67 [25]. According to the authors, digital image analysis of $\mathrm{PPH} 3$ or MAI combined with $\mathrm{Ki}-67$ is a valuable prognostic tool in the treatment of breast cancer patients with negative lymph nodes.

Discussing the merits of the use of currently available methods for histopathological SLN assessment, it is important to remember about the limited efficiency of these tests. Tan and colleagues from the Memorial Sloan Kettering Cancer Center in New York analyzed the data of 368 breast cancer patients, treated during the period 1976-1978 (mastectomy with axillary lymph node resection was performed; in the absence of nodal metastases, systemic therapy was not applied). Evaluation of axillary nodes performed again using IHC methods changed the earlier diagnoses in almost every fourth patient $(83 / 368$ - in $73 \%$ of cases ITC were detected, in $20 \%$ micrometastases, and in $6 \%$ metastases greater than $2 \mathrm{~mm}$ ) [26]. Therefore it is likely that the currently used SLN verification methods, including IHC techniques, might not meet the diagnostic requirements of future diagnostic methods.

\section{Conclusions}

Use of immunohistochemistry for diagnostics of sentinel lymph node metastases in patients with breast cancer enables detection of a greater proportion of metastases, thus modifying both surgical (SLN macrometastases) and adjuvant treatment. As compared to $\mathrm{pN} 0$ patients and with a metastasis found in HE staining, in patients with a metastasis in the sentinel lymph node diagnosed in IHC studies, no statistically significant differences were found concerning the long-term results of the implemented treatment.

The authors declare no conflict of interest.

\section{References}

1. Veronesi U, Banfi A, Del Vecchio M, et al. Comparison of Halsted mastectomy with quadrantectomy axillary dissection and radiotherapy in early breast cancer: long term results. Eur J Cancer Clin Oncol 1986; 22: 1085-1089.

2. Mansel RE, Fallowfield L, Kissin M, et al. Randomized multicenter trial of sentinel node biopsy versus standard axillary treatment in operable breast cancer: the ALMANAC Trial. J Natl Cancer Inst 2006; 98: 599-609.

3. Kim T, Giuliano AE, Lyman GH. Lymphatic mapping and sentinel lymph node biopsy in early-stage breast carcinoma: a metaanalysis. Cancer 2006; 106: 4-16.

4. Cserni G, Bianchi S, Vezzosi V, et al. Sentinel lymph node biopsy in staging small (up to $15 \mathrm{~mm}$ ) breast carcinomas. Results from a European multi-institutional study. Pathol Oncol Res 2007; 13: 5-14.

5. Zavagno G, De Salvo GL, Scalco G, et al. A randomized clinical trial on sentinel lymph node biopsy versus axillary lymph node dissection in breast cancer: results of the Sentinella/GIVOM trial. Ann Surg 2008; 247: 207-213.

6. Krag DN, Anderson SJ, Julian TB, et al. Sentinel-lymph-node resection compared with conventional axillary-lymph-node dissection in clinically node-negative patients with breast cancer: overall survival findings from the NSABP B-32 randomised phase 3 trial. Lancet Oncol 2010; 11: 927-933.

7. Veronesi U, Viale G, Paganelli G, et al. Sentinel lymph node biopsy in breast cancer. Ten-year results of a randomized controlled study. Ann Surg 2010; 251: 595-600.

8. Goldhirsch A, Wood WC, Coates AS and panel members: Strategies for subtypes-dealing with the diversity of breast cancer: highlights of the St Gallen International Expert Consensus on the Primary Therapy of Early Breast Cancer 2011. Ann Oncol 2011; 22: 1736-1747.

9. Martin RC 2nd, Edwards MJ, Wong SL, et al. Practical guidelines for optimal gamma probe detection of sentinel lymph nodes in breast cancer: results of a multi-institutional study. For the University of Louisville Breast Cancer Study Group. Surgery 2000; 128: 139-144.

10. Greene FL, American Joint Committee on Cancer, American Cancer Society, AJCC. Cancer Staging Manual, $6^{\text {th }}$ ed. Springer-Verlag, New York 2002.

11. Goldhirsch A, Wood WC, Gelber RD, et al. Progress and promise: highlights of the international expert consensus on the primary therapy of early breast cancer 2007. Ann Oncol 2007; 18: 1133-1144.

12. Funasako Y, Uenosono Y, Hirata M, et al. Utility of the GeneSearch breast lymph node assay for the rapid evaluation of sentinel lymph nodes in breast cancer. Cancer 2010; 116: 4450-4455.

13. Giobuin SM, Kavanagh DO, Myers E, et al. The significance of immunohistochemistry positivity in sentinel nodes which are negative on haematoxylin and eosin in breast cancer. Eur J Surg Oncol 2009; 35: 1257-1260. 
14. Carvalho SM, Mourão Netto M, Lima EN, et al. Sentinel node biopsy in breast cancer: results in a large series. Braz J Med Biol Res 2010; 43: 593-599.

15. Choi SH, Barsky SH, Chang HR. Clinicopathologic analysis of sentinel lymph node mapping in early breast cancer. Breast $\mathrm{J}$ 2003; 9: 153-162.

16. Carvalho MJ, Dias MF, Silva TS, et al. Breast cancer patients with micrometastases in sentinel lymph nodes: differences considering additional metastatic lymph nodes. Eur J Gynaecol Oncol 2009; 30: 631-634.

17. Pugliese M, Stempel M, Patil S, et al. The clinical impact and outcomes of immunohistochemistry-only metastasis in breast cancer. Am J Surg 2010; 200: 368-373.

18. Geertsema D, Gobardhan PD, Madsen EV, et al. Discordance of intraoperative frozen section analysis with definitive histology of sentinel lymph nodes in breast cancer surgery: complementary axillary lymph node dissection is irrelevant for subsequent systemic therapy. Ann Surg Oncol 2010; 17: 2690-2695.

19. Tille JC, Egger JF, Devillaz MC, et al. Frozen section in axillary sentinel lymph nodes for diagnosis of breast cancer micrometastasis. Anticancer Res 2009; 29: 4711-4716.

20. Blumencranz P, Whitworth PW, Deck K, et al. Scientific Impact Recognition Award. Sentinel node staging for breast cancer: intraoperative molecular pathology overcomes conventional histologic sampling errors. Am J Surg 2007; 194: 426-432.

21. Mansel RE, Goyal A, Douglas-Jones A, et al. Detection of breast cancer metastasis in sentinel lymph nodes using intra-operative real time GeneSearch BLN Assay in the operating room: results of the Cardiff study. Breast Cancer Res Treat 2009; 115: 595-600.

22. Tsujimoto M, Nakabayashi K, Yoshidome K, et al. One-step nucleic acid amplification for intraoperative detection of lymph node metastasis in breast cancer patients. Clin Cancer Res 2007; 13: 4807-4816.

23. Zhu L, Loo WT, Louis WC. PTEN and VEGF: possible predictors for sentinel lymph node micro-metastasis in breast cancer. Biomed Pharmacother 2007; 61: 558-561.

24. Hodorowicz-Zaniewska D, Kibil W, Małek A, et al. Evaluation of serum concentrations of vascular endothelial growth factor (VEGF) in breast cancer patients. Pol J Pathol 2012; 63: 25560

25. Gudlaugsson E, Klos J, Skaland I, et al. Prognostic comparison of the proliferation markers mitotic activity index, phosphohistone H3, Ki67, steroid receptors, HER2, high molecular weight cytokeratins and classical prognostic factors in $\mathrm{T}_{1-2} \mathrm{~N}_{0} \mathrm{M}_{0}$ breast cancer. Pol J Pathol 2013; 64: 1-8.

26. Tan LK, Giri D, Hummer AJ, et al. Occult axillary node metastases in breast cancer are prognostically significant: results in 368 node-negative patients with 20-year follow-up. J Clin Oncol 2008; 26: 1803-1809.

\section{Address for correspondence}

\section{Tomasz Nowikiewicz}

Oncology Center, Prof. Lukaszczyk Memorial Hospital

Prof. I. Romanowskiej 2

85-796 Bydgoszcz, Poland

e-mail: tomasz.nowikiewicz@gmail.com 